\title{
Study of serum magnesium levels in type I diabetes mellitus patients: a case control study
}

\author{
Chaudhary D. ${ }^{1}$, Narayanappa D. ${ }^{2}$ \\ ${ }^{1}$ Dr. Divya Chaudhary, Junior Resident, Department of Pediatric, ${ }^{2}$ Dr. Narayanappa D., Professor, Department of Pediatric, \\ both authors are affiliated with JSS Medical College, JSS Academy of Higher Education, Mysuru, Karnataka, India
}

Corresponding Author: Dr. Divya Chaudhary, Junior Resident, Pediatric Department, JSS Medical College, JSS Academy of Higher Education, Mysuru, Karnataka, India. E-mail: divyachaudhary19@gmail.com

\begin{abstract}
Introduction and background: Magnesium is involved in insulin synthesis. Some studies claim that there is hypomagnesemia in type I diabetes patients and they have implicated its use to increase insulin production, reduce the complications. This study was done to know serum magnesium levels in type I diabetes patients and whether there is any significant correlation between serum magnesium levels and glycemic control. As there are variety of results found in different studies it was important to evaluate serum magnesium levels in type I DM patients and any scope of improvement of insulin levels by its supplementation. Materials and Methods: A case control study was done involving 44 cases, 44 sex and age matched controls. All patients less than 18yrs diagnosed with type I diabetes mellitus were included in study. Serum magnesium levels in cases and controls were compared. Percentage of hypomagnesemia in both groups, correlation between HbA1c levels and serum magnesium levels in cases were calculated. Results: $27.27 \%$ of type I DM whereas $11.4 \%$ of controls had hypomagnesemia. Mean serum magnesium level in cases and normal controls were 1.83 and 1.88 respectively (p 0.8 ). Conclusion: Hypomagnesemia in cases was more than double as compared to controls. Although, there was no significant difference between mean serum magnesium levels in cases and controls, no significant correlation between serum magnesium and $\mathrm{HbA} 1 \mathrm{C}$ levels was found in cases. Therefore, magnesium supplementation in type I diabetes mellitus for improvement of insulin levels is not recommended.
\end{abstract}

Keywords: HbA1c, Hypomagnesemia, Insulin, Magnesium, Type I Diabetes Mellitus

\section{Introduction}

Type I diabetes is a chronic disease, causing significant morbidity in childhood. It has impact on health but also on lifestyle and psychosocial development of a child.

Magnesium, the second most common intracellular cation, plays a fundamental role as a cofactor in various enzymatic reactions involving energy metabolism, glucose utilization by cells. It is also involved at multiple levels in insulin synthesis, secretion, binding and activity. Magnesium deficiency can alter the activity of membrane bound sodium potassium ATPase which is involved in maintenance of sodium potassium gradient across cells and also glucose transport.

Hypomagnesemia has been related as a cause of insulin resistance, and when it is chronic, it leads to the installation of macro and microvascular complications of diabetes [1].

Manuscript received: $\mathbf{1 0}^{\text {th }}$ December 2019

Reviewed: $\mathbf{2 0}^{\text {th }}$ December 2019

Author Corrected: $\mathbf{2 6}^{\text {th }}$ December 2019

Accepted for Publication: 31 ${ }^{\text {st }}$ December 2019
The mechanism involving diabetes mellitus and hypomagnesemia is still unclear, although some metabolic studies demonstrate that $\mathrm{Mg}$ supplementation has a beneficial effect in the action of insulin and in the glucose metabolism. Experimental studies have shown that hypomagnesemia inhibits prostacyclin receptor function, producing an imbalance between prostacyclin and thromboxane effects.

Hypomagnesemia can increase platelet reactivity, increase vascular and adrenal responses to angiotensin II, enhance thromboxane A 2 (TXA 2) release, and leads to organ damage from free radicals [2]. To date, the precise mechanism for the development of diabetic macrovascular changes is not clearly explained. Since the risk of ischemic heart disease is increased in diabetes, magnesium depletion may be one of the contributing factors to the development of ischemic heart disease in diabetics [3].

Many studies have claimed the importance of hypomagnesemia and scope of magnesium supplementation in 
improvement of insulin synthesis and reduction of complications associated with diabetes [4] whereas some studies have declined existence of hypomagnesemia in these patients [5].

Therefore, there is a need to find out magnesium levels in diabetes and to interrogate any correlation between serum magnesium levels and glycemic control but no such study has been done in India. Any future prospect of magnesium supplementation in these patients can open gates for new treatment options.

\section{Methods}

Type of Study and Setting: A case control study was done in hospitalized setup from Oct 2015 to August 2018.

Sampling methods: $3 \mathrm{ml}$ of blood sample was taken by fresh venupuncture and serum magnesium levels estimated by xylidyl dye method in both controls and cases after taking valid consent.

Sample size calculation: 44 cases and 44 controls were included; sample size was calculated based on incidence and prevalence of type I diabetes mellitus in the area.

\section{Inclusion criteria}

Patients with type I diabetes mellitus (<18years), admitted or coming as outpatient, diagnosis confirmed by $\mathrm{C}$ peptide levels, were included as cases. Age and sex matched controls were taken.

\section{Exclusion criteria}

Other conditions affecting serum magnesium levels chronic diarrhoea, malnutrition, malabsorption, renal failure, acute pancreatitis, patients on diuretics, asthma, seizures/epilepsy, patients on magnesium containing antacids.

Data collection procedure: After taking informed consent from parents of study subjects, questionnaire was asked to obtain history and thorough physical examination was done. Magnesium reacts with xylidyl blue to form a coloured compound in alkaline solution. The intensity of colour formed is proportional to the magnesium in sample estimated by colorimetric method, normal serum magnesium level was taken as $1.7-2.7 \mathrm{mg} / \mathrm{dl}$ according to the method used in estimation.

Ethical consideration and permission: Ethical committee clearance was taken at the institute in year 2015 , before starting the study.

Data Analysis: The data collected was entered in MS excel 2010 and analyzed using SSPS version 22 . Descriptive statistical measures like percentage, mean, standard deviation were applied. Inferential statistical tests like independent sample t test, chi square test, Pearson's correlation were applied. Serum magnesium levels of cases and controls were compared, percentage of hypomagnesemia in both groups was calculated. In cases correlation between serum magnesium levels and hypoglycemic control (HbA1c levels) was calculated.

\section{Results}

In the present study, $40.9 \%$ of cases were in age group $0-9$ years, $59.1 \%$ of cases were in group $10-18$ years. $38 \%$ of controls were in group 0-9years, whereas 62\% were in group 10-18years. Median age of cases was 12 years, median age of 44 controls was 10.5 yrs.

There was almost equal sex distribution in cases and controls, $52.3 \%$ males that is 23 out of $44,47.7 \%$ females that is 21 out of 44. Mean duration of treatment was $1.8 y e a r s$, both newly diagnosed and previously treated cases were included in the study.

Mean insulin treatment received was 1.06 units/kg/day. $12(27.27 \%)$ cases had hypomagnesemia as compared to 5 (11.4\%) of controls (Table 1). I

Table-I: Hypomagnesemia in cases and controls

\begin{tabular}{|c|c|c|}
\hline Magnesium level & Cases & Controls \\
\hline Normal & $72.73 \%$ & $88.6 \%$ \\
\hline Hypomagnesemia & $27.27 \%$ & $11.4 \%$ \\
\hline
\end{tabular}

*Out of 44 cases, 12(27.27\%) had hypomagnesemia. Out of 44 controls 5(11.4\%) had hypomagnesemia.

Mean serum magnesium values in cases and the control group were $1.83(0.36)$ and $1.88(0.26)$ (Table 2). There was no significant difference in serum magnesium levels of cases and controls, $\mathrm{p}$ value being 0.8 . 
Original Research Article

Table-2: Mean serum magnesium levels in cases and controls.

\begin{tabular}{|c|c|c|}
\hline \multirow{2}{*}{} & \multicolumn{2}{|c|}{ Magnesium } \\
\cline { 2 - 3 } & Mean & Standard Deviation \\
\hline Cases & 1.83 & .36 \\
\hline Control & 1.88 & .26 \\
\hline
\end{tabular}

* Mean serum magnesium level in cases and normal controls are 1.83 with standard deviation of 0.36 and 1.88 standard deviation 0.26 respectively.

\# p value of 0.8 which is more than 0.05 therefore there is no significant correlation between serum magnesium levels and type I DM.

Mean HbA1C levels in cases with normal magnesium was $12.34 \pm 1.81$ and in cases with hypomagnesemia was $11.99 \pm 3.05$. There was no significant correlation between serum magnesium levels and HbA1C levels, p value being 0.23 (Table 3 ).

Table-3: Correlation between cases with hypomagnesemia and HbA1C levels.

\begin{tabular}{|c|c|c|c|}
\hline \multirow{2}{*}{ Cases } & \multicolumn{2}{c|}{ HbA1C } \\
\cline { 3 - 4 } & Normal & Mean & Standard Deviation \\
\hline \multirow{2}{*}{ Magnesium } & Low & 12.34 & 1.81 \\
\cline { 2 - 4 } & & 11.99 & 3.05 \\
\hline
\end{tabular}

*Mean HbA1C levels in cases with normal magnesium was 12.34 with standard deviation of 1.81 and in cases with hypomagnesemia was 11.99 with standard deviation of 3.05 .

\# p value being 0.23 .

\section{Discussion}

The term diabetes mellitus describes a complex metabolic disorder characterized by chronic hyperglycemia resulting from defects in insulin secretion, insulin action, or both. Inadequate insulin secretion and/or diminished tissue responses to insulin in the complex pathways of hormone action result in deficient insulin action on target tissues, which leads to abnormalities of carbohydrate, fat, and protein metabolism [6].

It is estimated that India is housing about 97,700 children with type 1 diabetes mellitus (T1DM). A study of 30 children with insulin-dependent diabetes with age at diagnosis $\leq 15$ years, conducted in 1992, reported a prevalence of $0.26 / 1000$ children. The peak age at diagnosis was 12 years. The Karnataka state T1DM registry listed an incidence of 3.7/100,000 in boys and 4.0/100,000 in girls over 13 years of data collection [7].

$\mathrm{Mg}$ is the second predominant component in the intracellular compartment, an important regulator of the cellular processes, co-factor of more than 300 essential metabolic reactions, including the ones that produce or use the Mg ATP complex. Its functionality is related to the synthesis of tissue constituents, growth and thermogenesis, and with the activity of tyrosine kinase, in the metabolism of glucose [8]. The homeostasis of $\mathrm{Mg}$ depends on the amount of ingestion, efficiency of absorption and on the intestinal and renal excretion.
The absorption of this mineral occurs along the whole intestinal tract, especially in the ileum and colon, and its regulation is made mainly by the kidney [9]. The responsible mechanisms for $\mathrm{Mg}$ deficiency in patients with diabetes have still not been clarified. It is speculated that one of the triggering factors is the increase in urinary excretion of $\mathrm{Mg}$, especially in patients with unsatisfactory metabolic control, dietary deficiency or absorption reduction. The scientific evidences indicate the role of the $\mathrm{Mg}$ as mediators of the insulin action. In diabetes occur chronic alterations of homeostasis of intracellular $\mathrm{Mg}$. The effects result in damages on the tyrosine kinase activity, in level of receptors unchaining the outlying insulin synthesis and resistance [10].

The hyperglycemia, independent of insulin, or other responses due to oral glucose ingestion has potential role in cellular ionic changes of free $\mathrm{Ca}$ and $\mathrm{Mg}$. The hyperglycemia per se elevated intracellular $\mathrm{Ca}$ and suppressed intracellular $\mathrm{Mg}$ in normal human red cells. The $\mathrm{Mg}$ deficit interferes on enzymatic reactions that use or produce adenosine triphosphate (ATP), in consequence modifies the enzymatic cascade that involves the carbohydrates metabolism, triggering diabetes. As observed by Delva et al, [11] in hyperglycemia, existing ionic alterations in the cells, there is an increase of the intracellular ATP concentration, that induce a reduction of the intracellular ionized $\mathrm{Mg}$ levels. 
In patients with diabetes, the supplementation with micronutrients must be individualized, based on dietary history, clinical features and laboratorial evaluation of the nutritional state [12]. Even so the oral supplementation with $\mathrm{Mg}$ improves the secretion and sensitivity to insulin in type 1 and 2 diabetic patients, there are still controversies based on human and animal studies accomplished.

In the present study, out of 44 cases, $27.27 \%$ had hypomagnesemia whereas out of 44 controls only 5 (11.4\%) had hypomagnesemia. By our case-control study involving 44 cases and 44 controls, no significant correlation was found between serum magnesium level and type I DM, therefore, questioning any future role of magnesium supplementation on improvement of serum insulin levels. Similar study was done in Taiwan by Ching Chiang Lin et al in 2015 on 88 patients, where only $3.4 \%$ of diabetes patients were found to have hypomagnesemia, in addition they have found that low serum magnesium levels correlate with high malondialdehyde levels $(p<0.001$, which is a marker of oxidative stress [13].

Vahap et al have done study in Turkey (2016) on intra erythrocytic levels of magnesium in type I DM patients which was found to be same in cases and controls, whereas intracellular retention of magnesium and urinary excretion of magnesium were increased after IV administration of magnesium challenge $90.2 \%$ as compared to $50 \%$ in controls, therefore depicting intracellular deficiency of magnesium [14].

On the other hand study done in Egypt on 25 type I DM patients had shown significantly low serum magnesium levels in cases as compared to controls, also they have found negative correlation between duration of illness and serum magnesium levels. A study done in Egypt observed that the relation between serum magnesium levels and glycemic control, on 150 type I DM patients, it was observed that $37.3 \%$ of patients had hypomagnesemia and those patients had higher $\mathrm{HbA1C}$ levels as compared to rest [15].

A study done by Doaaa Shahbah et al in 2017, in Egypt, on 71 patients, has found hypomagnesemia in $28.2 \%$ patients, they have also found that patients with lower magnesium levels had higher HbA1C, LDL, total cholesterol, triglyceride levels, therefore correlating serum magnesium level to complications of diabetes. Also, they have supplemented the patients with hypomagnesemia with oral magnesium for 3 months and they found significant improvement in HbA1C levels [16].

In a cross-section study done on 50 type I and type II diabetes mellitus patients, in 2007, in Bikaner it was found that diabetic patients have significant hypomagnesemia $(\mathrm{p}<0.005)$, Serum magnesium was significantly low in diabetes with complication than without complications ( $p$ $<0.001)$. Duration of diabetes and serum magnesium were inversely related. Poor glycaemic control was associated with hypomagnesaemia $(-2.623 ; \mathrm{p}<0.05)$. There was strong association between hypomagnesaemia and retinopathy $(1.76 \pm 0.26)$, obesity $(1.878 \pm 0.326)$ and hypertension $(1.75 \pm 0.071)$ and it was statistically significantly ( $p<0.005,0.042,0.000$ respectively). Hence it was concluded that the change in serum magnesium level may have a bearing on the complication and morbidity in patients of diabetes mellitus [1].

Limitation of this study- Intra erythrocytic magnesium level is more accurate as compared to serum magnesium level as magnesium is mainly intracellular ion.

\section{Conclusion}

As there are variety of results found in different studies it was important to evaluate serum magnesium levels in type I DM patients and any scope of improvement of insulin levels by $\mathrm{Mg}$ supplementation in future should be looked into. Although hypomagnesemia in type I diabetes mellitus patients is more than controls but magnesium level is not significantly reduced. Hence, hypomagnesemia cannot be implicated as contributory factor in insulin reduction and complications in diabetes. Magnesium supplementation in diabetes therefore, may not be useful. Intra erythrocytic magnesium level is more accurate as compared to serum magnesium level as magnesium is mainly intracellular ion, therefore measurement of intracellular magnesium levels in type I diabetes patients may give more information about its status in these patients.

\section{What this study adds to the existing knowledge?}

Magnesium is not significantly low in type I DM patients as compared to controls. Therefore, supplementation of magnesium in these patients is not recommended. Hypomagnesemia does not correlate with HbA1c levels, there is no correlation between severity of disease and hypomagnesemia.

\section{Author's contribution}

Dr. Divya Chaudhary: Design of study, acquisition and analysis of data and drafting the manuscript.

Dr. Narayanappa D.: Contributed in study design, interpretation of data, revising and final approval of article.

Funding: No funding sources

Conflict of interest: None declared 
Original Research Article

Ethical Approval: This study was approved by the Institutional Ethics Committee

\section{References}

1. Sharma A, Dabla S, Agrawal RP, Barjatya H, Kochar DK, Kothari RP. Serum magnesium: an early predictor of course and complications of diabetes mellitus. J Ind Med Assoc. 2007;105(1):16-20.

2. Wegner M, Araszkiewicz A, Zozulińska Ziółkiewicz D, Wierusz Wysocka B, et al. The relationship between concentrations of magnesium and oxidized low-density lipoprotein and the activity of platelet activating factor acetylhydrolase in the serum of patients with type 1 diabetes. Mag Res J. 2010;23(2):97-104. doi: https:// www.jle.com/10 1684/mrh.2010.0207.

3. Shechter M. The role of magnesium as antithrombotic therapy. Wiener Medizinische Wochenschrift. 2000;150 (15-16):343-347.

4. Galli-Tsinopoulou A, Maggana I, Kyrgios I, Mouzaki K, Grammatikopoulou MG, Stylianou C et al. Association between magnesium concentration and $\mathrm{HbA} 1 \mathrm{c}$ in children and adolescents with type 1 diabetes mellitus. J Diab. 2014;6 (4):369-377. doi: https://doi.org/10.1111/17530407. 12118.

5. Derakhshan R, Balaee P, Darakhshan S, Masoodpoor N, Banihosseini SS. Lipid profile, thyroid function, and serum magnesium level in type I diabetic children. Minerva Pediatrica. 2011;63(1):27-33.

6. World Health Organisation. Definition and Diagnosis of Diabetes Mellitus and Intermediate Hyperglycaemia: Report of a WHO/IDF Consultation. Geneva, Switzerland: World Health Organisation, 2006. Available at: https:// www.who.int/diabetes/publications/Definition $\% 20$ and $\% 2$ 0diagnosis\%20of\%20diabetes_new.pdf.

7. Kanakatte Mylariah Prasanna K. Incidence trends for childhood type 1 diabetes in India. Indian $\mathrm{J}$ Endocrinol Metabol. 2015;19 (1):34-35. doi: https://doi.org/10.4103/ 2230-8210.155378.

8. Standing Committee on the Scientific Evaluation of Dietary Reference Intakes; Food and Nutrition Board;
Institute of Medicine. Dietary reference intakes for calcium, phosphorus, magnesium, vitamin $\mathrm{D}$, and fluoride. Washington, DC: National Academy Press; 1997;6:190249. doi: https://doi.org/10.17226/5776.

9. Valk HW. Magnesium in diabetes mellitus. Netherlands J Med. 1999; 54:139-146. doi: https://doi.org/10.1016/ S0300-2977(99)00005-4.

10. Sales CH, Pedrosa Lde F. Magnesium and diabetes: their relation. Clinic Nutri J.2006;25(4):554-562. doi: https://doi.org/10.1016/j.clnu.2006.03.003.

11. Delva P, Degan M, Pastori C, Faccini G, Lechi A. Glucose induced alterations of intracellular ionized magnesium in human lymphocytes. Life Sci. 2002;71 (18):2119-2135. doi: https://doi.org/10.1016/ S0024 3205(02) 01992-6.

12. Mooradian AD, Failla M, Hoogwerf B, Maryniuk M, Wylie Rosett J. Selected vitamins and minerals in diabetes. Diabetes Care. 1994;17(5):464-479. doi: https:// doi.org/10.2337/diacare.17.5.464.

13. Ching-Chiang L, Guey-Ju T, Cheng-Fa L, Bai-Hsiun C, Yeou-Lih H. Magnesium, zinc, and chromium levels in children, adolescents, and young adults with type 1 diabetes. Clinic Nutri. 2016;35(4):880-884. doi: https:// doi.org/10. 1016/j.clnu.2015.05.022.

14. Vahap U, Cigdem B, Enver S, Cengiz B. Cellular trace element changes in type 1 diabetes patients. J Clinic Res Pediatr Endocrinol. 2016;8(2):180-186. doi: https:// doi.org/ 10.4274/jcrpe. 2449 .

15. Asmaa MN, Samira SZ, Aliaa MM, Bassem HG. The relationship between hypomagnesaemia and glycemic control in children with type 1 diabetes mellitus. J Diabetes Metabol Disord. 2016;7(8):1000693. doi: https://doi.org/10.4172/2155-6156.1000693.

16. Shahbah D, Hassan T, Morsy S, Saadany HE, Fathy M, Al-Ghobashy A et al. Oral magnesium supplementation improves glycemic control and lipid profile in children with type 1 diabetes and hypomagnesaemia. Med (Baltimore). 2017; 96 (11):e 6352. doi: https:// doi. org/10.1097/MD. 0000000000006352.

\section{How to cite this article?}

Chaudhary D., Narayanappa D. Study of serum magnesium levels in type I diabetes mellitus patients: a case control study. Int J Pediatr Res.2019; 6(12):620-624.doi:10.17511/ijpr.2019.i12.06 